This copy is for your personal, non-commercial use only.

If you wish to distribute this article to others, you can order high-quality copies for your colleagues, clients, or customers by clicking here.

Permission to republish or repurpose articles or portions of articles can be obtained by following the guidelines here.

The following resources related to this article are available online at www.sciencemag.org (this information is current as of December 6, 2013 ):

Updated information and services, including high-resolution figures, can be found in the online version of this article at:

http://www.sciencemag.org/content/342/6163/1251.full.html

Supporting Online Material can be found at:

http://www.sciencemag.org/content/suppl/2013/12/04/342.6163.1251.DC1.html

A list of selected additional articles on the Science Web sites related to this article can be found at:

http://www.sciencemag.org/content/342/6163/1251.full.html\#related

This article cites $\mathbf{3 0}$ articles, 10 of which can be accessed free:

http://www.sciencemag.org/content/342/6163/1251.full.html\#ref-list-1 


\section{Intact But Less Accessible Phonetic Representations in Adults with Dyslexia}

\author{
Bart Boets, ${ }^{1,2 *}$ Hans P. Op de Beeck, ${ }^{3}$ Maaike Vandermosten, ${ }^{2}$ Sophie K. Scott, ${ }^{4}$ \\ Céline R. Gillebert, ${ }^{5}$ Dante Mantini, ${ }^{5,6}{ }^{\text {Jessica Bulthé, }}{ }^{3}$ Stefan Sunaert, ${ }^{7}$ \\ Jan Wouters, ${ }^{8}$ Pol Ghesquière ${ }^{2}$
}

Dyslexia is a severe and persistent reading and spelling disorder caused by impairment in the ability to manipulate speech sounds. We combined functional magnetic resonance brain imaging with multivoxel pattern analysis and functional and structural connectivity analysis in an effort to disentangle whether dyslexics' phonological deficits are caused by poor quality of the phonetic representations or by difficulties in accessing intact phonetic representations. We found that phonetic representations are hosted bilaterally in primary and secondary auditory cortices and that their neural quality (in terms of robustness and distinctness) is intact in adults with dyslexia. However, the functional and structural connectivity between the bilateral auditory cortices and the left inferior frontal gyrus (a region involved in higher-level phonological processing) is significantly hampered in dyslexics, suggesting deficient access to otherwise intact phonetic representations.

$\mathrm{S}$ peech perception involves the mapping of spectrally complex and rapidly changing acoustic signals onto discrete and abstract phonetic sound categories or phonemes (1). Developmental dyslexia is a hereditary neurological disorder characterized by severe and persistent reading and/or spelling impairments (2). Individuals with dyslexia perform poorly on tasks that require phonological awareness, verbal short-term memory, and lexical access. Performance on these phonological tasks predicts reading acquisition in both normal and dyslexic readers (3). One view is that success on these tasks reflects the quality of underlying phonological (phonetic) representations (4) and that these representations of speech sounds are distorted or less well specified in individuals with dyslexia (5). An alternative view holds that in people with dyslexia, representations are intact but access to the representations is problematic $(6,7)$. Here, we combined functional magnetic resonance imaging (fMRI) with multivoxel pattern analysis (MVPA) $(8-10)$ and functional and structural connectivity analysis to disentangle whether dyslexia is caused by poor quality of the phonetic representation or by difficulty in accessing an intact representation.

We collected whole-brain functional images in 23 adults with a diagnosis of dyslexia and 22 matched normal readers (table S1) (11-13) while they listened to different versions of four

\footnotetext{
${ }^{1}$ Child and Adolescent Psychiatry, KU Leuven, 3000 Leuven, Belgium. ${ }^{2}$ Parenting and Special Education Research Unit, KU Leuven, 3000 Leuven, Belgium. ${ }^{3}$ Laboratory of Biological Psychology, KU Leuven, 3000 Leuven, Belgium. ${ }^{4}$ Institute of Cognitive Neuroscience, University College London, London WC1N 3AR, UK. ${ }^{5}$ Department of Experimental Psychology, University of Oxford, Oxford OX1 3UD, UK. ${ }^{6}$ Department of Health Sciences and Technology, ETH Zürich, 8092 Zürich, Switzerland. ${ }^{7}$ Department of Radiology, KU Leuven, 3000 Leuven, Belgium. ${ }^{8}$ ExpORL, Department of Neurosciences, KU Leuven, 3000 Leuven, Belgium.

*Corresponding author. E-mail: bart.boets@ppw.kuleuven.be
}

sublexical speech sounds (fig. S1) and performed an easy phoneme discrimination task. The selection of stimuli allowed us to investigate both vowel and stop-consonant discrimination, which rely on spectral and spectrotemporal acoustic feature processing, respectively. If dyslexia is related to a deficit in the quality of phonetic representations, then we expect that the neural representations would be less robust and distinct in individuals with dyslexia than in normal readers. Given dyslexics' particular problems processing temporal cues, such as those involved in consonant discrimination (11), we expected the most prominent group differences for neural patterns distinguishing between consonants.

We analyzed the pattern of multivoxel activity within six left-hemisphere and six right-hemisphere regions involved in speech processing and within one non-speech control region (primary visual cortex V1) (table S2) $(8,14)$. For each of these regions, we correlated the activity pattern in response to each stimulus in one-half of the data with the activity pattern in response to each stimulus in the remaining data (fig. S2). Figure 1 displays averaged correlations as a function of the phonetic similarity of the pairs: phonetically identical, differing in consonant, differing in vowel, and differing in both consonant and vowel. Comparison of these correlations indicates to what extent various acoustic realizations of the same phoneme elicit a similar activation pattern while different phonemes elicit a distinct activation pattern. We found significant differences between the four phonetic comparisons in bilateral primary auditory cortex (PAC), superior temporal gyrus (STG), middle temporal gyrus (MTG), and supramarginal gyrus $(\mathrm{SMG})(P \mathrm{~s}<0.0003)$, and unilaterally in right angular gyrus (AG) and right inferior frontal gyrus $(\mathrm{IFG})\left(P_{\mathrm{S}}<0.03\right)$ [repeated-measures analyses of variance (ANOVAs) with group as between-subject factor and phonetic compari- son as within-subject factor]. We found no differences between phonetic comparisons for left AG and left IFG and for area V1 $(F<1)$. Activity patterns were equally reliable in both groups, except for right SMG, where the neural representations of the dyslexic readers were significantly more distinct than those of the normal readers (group $\times$ comparison interaction: $P=0.024$ ) . Focusing on the most crucial comparison entailing temporal cues, we observed that speech sounds differing in consonant could be differentiated in left PAC, STG, MTG, and SMG $\left(P_{\mathrm{S}}<0.02\right.$; for all other regions, $P \mathrm{~S}>0.16)$. Activity patterns differentiating between consonants were equally reliable in both groups (all group $\times$ comparison interactions: $P>0.28$ ), except for right STG, where differentiation between consonants was feasible for dyslexic $(P=0.037)$ but not for normal readers $(P=$ 0.977). Across all regions and for both reading groups, left-hemisphere regions were significantly more sensitive than right-hemisphere regions to differences in consonants $(P=0.017)$ [consistent with the left-hemisphere bias for temporal cues as described in the literature $(10,15)]$. We found no lateralization for vowel decoding in either normal or dyslexic readers $(F<1)$.

To ensure that we did not overlook any brain region hosting superior representations in normal as compared to dyslexic readers, we performed a whole-brain searchlight MVPA (16). A spherical "searchlight" was moved across the entire brain, and for every local region we calculated how well the response patterns differentiated between speech sounds. This analysis confirmed that phonetic representations are primarily hosted bilaterally in primary and secondary auditory cortices and that both normal and dyslexic readers shared similar quality of these representations (fig. S4).

Thus, we have no indication of poorer quality of phonetic representations in dyslexic readers. The MVPA results show that phonetic representations of dyslexic readers were at least as robust and distinct as those of normal readers. It may be that dyslexic readers achieve normal neural representations through greater than normal effort. Indeed, on the phoneme discrimination task administered during scanning, dyslexic readers achieved normal accuracy (as such, we avoided the issue that findings pertaining to brain activity may be confounded by differences in accuracy), but at slower speed (table S1). Attention may modulate brain activity and alter brain activity profiles (17). To investigate phonetic representations under conditions that elicit less compensational processing, we recalculated the previous analyses using only the activity pattern in response to speech sounds that were less relevant for the task at hand (the third speech sound in a stimulus block of four). As these stimuli were processed less intentionally, they yielded less brain activity (table S3). Nonetheless, even under this more stringent condition, speech sounds could be differentiated in left and right PAC, STG, MTG, and SMG $(P \mathrm{~s}<0.05)$. And again, although dyslexic readers overall presented less activation 
(table S3), the quality of the phonetic representations was equal in both groups (all group $\times$ comparison interactions: $P>0.25$ ) (fig. S5).

Thus, we found no difference in the neural quality of phonetic representations between dyslexic and normal readers. Although we cannot rule out that dyslexics' neural representations may have been less well specified at a younger age or would follow a different temporal trajectory detectable through techniques such as electroencephalography (18), our results indicate that the phonetic representations can be intact in adult dyslexics despite persisting reading difficulties $(6,7)$. Therefore, we sought to investigate the alternative hypothesis of impaired access to phonetic representations.

Several studies have shown that Broca's area, particularly the left IFG pars opercularis, is involved in sensory-motor integration and effortful phonological processing $(19,20)$. Hence, this area, which itself does not host phonetic representations, must access the representations in primary and secondary auditory cortices to compute the required phonological manipulations. We investigated the efficiency of access or the quality of interregional brain communication by assessing intrinsic functional connectivity between each possible pairing of the 13 anatomical regions shown in Fig. 1 (21). In each region we selected the most active cluster during task performance, and we calculated the correlations between the residualized signal intensity time series of each pair of these 13 predefined seed regions (Fig. 2) (22). Both groups showed equally strong connectivity among bilateral temporal areas (primary and secondary auditory cortices) across both hemispheres. Bilateral temporal areas were functionally connected with left IFG, but this connection was smaller in the dyslexic group, in particular for left STG and right PAC $(P \mathrm{~s}<0.005$, corrected for multiple comparisons). Without multiple testing correction, the group difference in functional connectivity between left IFG and right STG $(P=$ 0.067 ) and between left IFG and right MTG (near to superior temporal sulcus) $(P=0.051)$ also approached significance. Individual differences in the strength of functional connectivity between left STG and left IFG correlated with behavioral indices of word reading $(r=0.40)$, nonword reading $(r=0.48)$, spelling $(r=0.53)$, phonological awareness $(r=0.46)$, verbal short-term memory $(r=0.44)$, and lexical access $(r=0.46)$, as well as with reaction time on the phoneme discrimination task performed in the scanner $(r=-0.51)$ (all $P_{\mathrm{s}}<0.01$ ).

At a neuroanatomical level, adequate communication between left IFG and left STG is effected by the left arcuate fasciculus, the major language tract that ensures an efficient signal transmission between Wernicke's and Broca's areas. We recently collected diffusion tensor imaging data in a subsample $(N=32)$ of our participants and delineated the left arcuate fasciculus and its three constituent segments (direct, anterior, and posterior) on the basis of whole-brain
Fig. 1. Quality of phonetic representations as derived from multivoxel pattern analysis. Average correlations between the (normalized) activity patterns elicited by phonetically identical syllables, syllables differing in consonant, syllables differing in vowel, and syllables differing in both consonant and vowel for dyslexic (DR) and normal readers (NR) in each of the anatomical regions; error bars represent SE. The larger the overall quality of the phonetic representations, the larger the differences between the baseline correlation (between phonetically identical syllables) and the other correlations. Correlations differing from this baseline correlation are indicated with blue asterisks (paired $t$ tests) and green asterisks (repeated-measures ANOVA, Tukey-corrected post hoc $t$ tests) $\left({ }^{*} P<0.05\right.$, ${ }^{* \star P}<0.01$, ${ }^{\star \star \star} P<0.001$, $\star * * * P<0.0001)$. Images at left are representations of the left hemisphere anatomical regions.
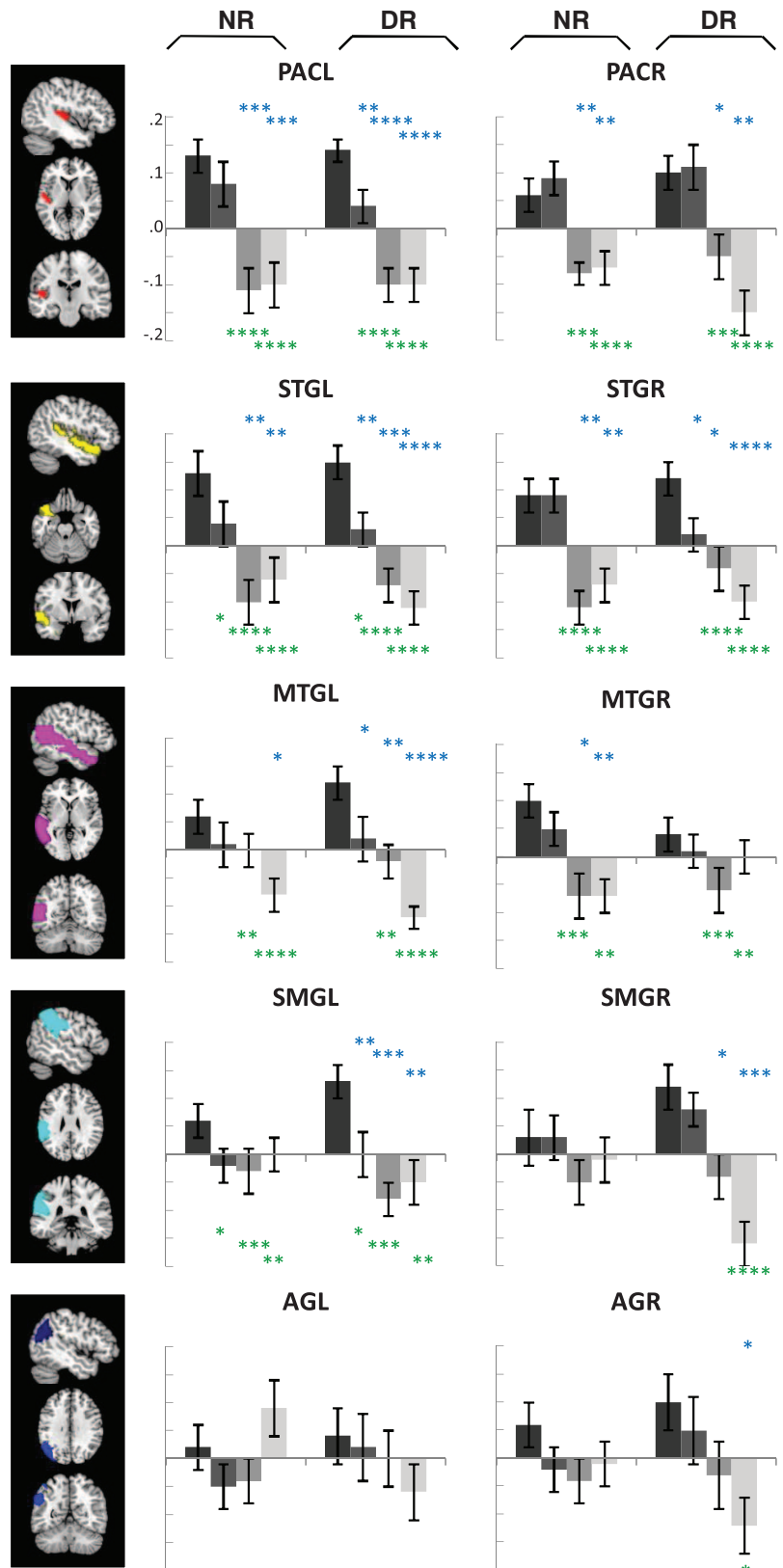

AGL

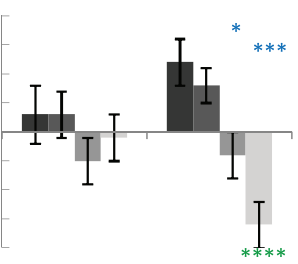

AGR

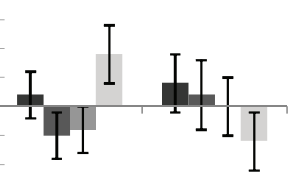

IFGL
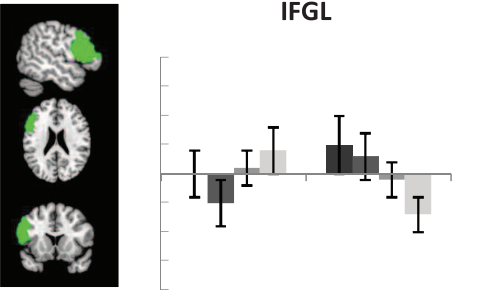

V1
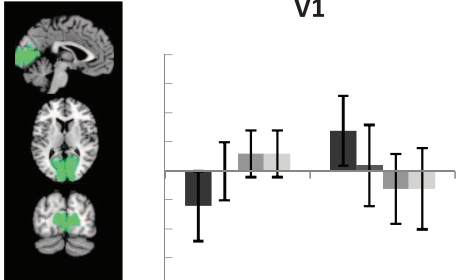

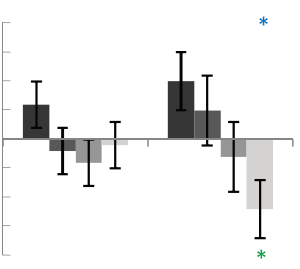

IFGR

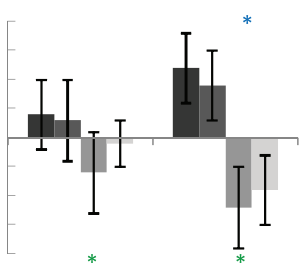

- Phonetically identical

口 Different consonant

口 Different vowel

Different consonant and vowel 
A
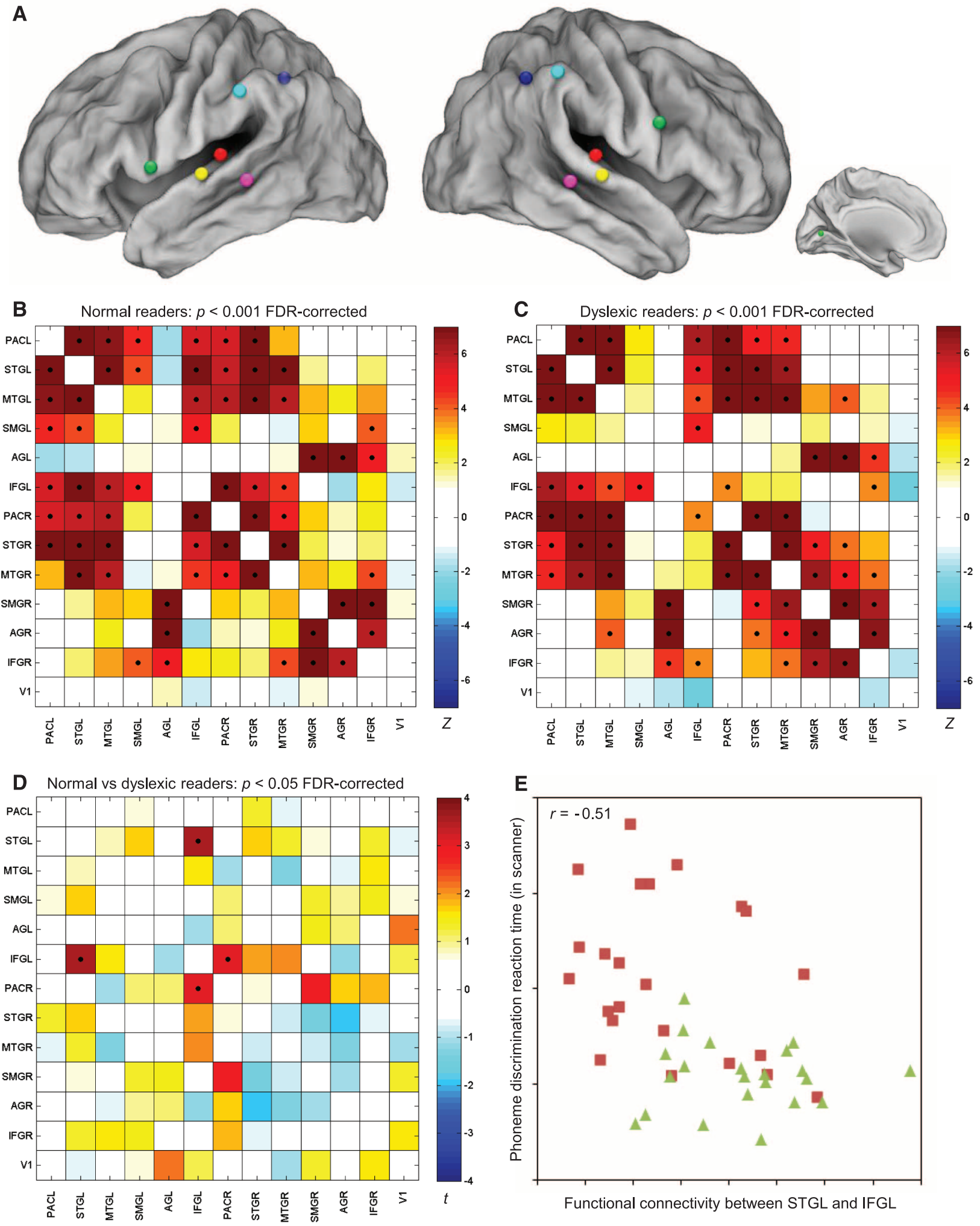

Fig. 2. Functional connectivity analysis. (A) Schematic representation of the predefined seed regions. The color coding corresponds with that used in Fig. 1. (B and C) Color-coded matrices represent functional connectivity (expressed as $Z$ scores) among the 13 seed regions in normal and dyslexic readers. Significant correlations $(P<0.001$, false discovery rate-corrected) are indicated by a black dot. (D) Statistical comparison of the functional

connectivity between the groups. Significant group differences in functional connectivity are indicated by a black dot (independent $t$ test, $P<0.05$, false discovery rate-corrected). (E) Scatterplot of the association between reaction time on the phoneme discrimination task in the scanner ( $y$ axis) and intrinsic functional connectivity between left STG and left IFG ( $x$ axis). Dyslexic readers are depicted by red squares, normal readers by green triangles. 
tractography (12). For the present report, we complemented these data and analyses to comprise the full sample $(N=45)$. Group comparisons (table S1) revealed significantly reduced white matter integrity of the left arcuate fasciculus in dyslexics $(P=0.019)$, in particular in the segment that directly connects posterior temporal and frontal areas $(P=0.038)$; this result provides neuroanatomical evidence that corroborates the deficiency in functional connectivity between left IFG and left STG. The functional and structural connectivity measures were not mutually related $(r=0.06, P=$ $0.70)$. This is in line with recent evidence highlighting the differences between the two types of connectivity measures $(21,23)$ and suggests that both measures are complementary, each capturing a different aspect of the communication between left frontal and temporal language areas. Together, the functional and structural connectivity measures accounted for $35 \%$ of the variance in reading and spelling ability, and they predicted reading status (dyslexic versus normal reader) with an accuracy of $73 \%$. This finding adds to the growing recognition of dyslexia as a disconnection syndrome $(24,25)$.

Our results indicate that deficient phonetic representations are not the core problem in dyslexia. Does this imply that it is time to abandon the influential phonological deficit hypothesis? No, certainly not. The behavioral data of our dyslexic participants reveal that they do show severe deficits in the traditional phonological domains, including phonological awareness, verbal short-term memory, and lexical access (table S1) $(11,13)$. Yet our neuroimaging findings suggest that it is not a deficit in underlying representations that characterizes dyslexia. Instead, our results suggest that a dysfunctional connection between frontal and temporal language areas impedes efficient access to otherwise intact representations of speech sounds, thus hampering a person's ability to manipulate them fluently.

\section{References and Notes}

1. E. F. Chang et al., Nat. Neurosci. 13, 1428-1432 (2010).

2. F. R. Vellutino, J. M. Fletcher, M. J. Snowling, D. M. Scanlon, J. Child Psychol. Psychiatry 45, 2-40 (2004).

3. B. Boets et al., Br. J. Dev. Psychol. 28, 5-31 (2010)

4. R. K. Wagner, J. K. Torgesen, Psychol. Bull. 101 192-212 (1987)

5. U. Goswami, Dyslexia 6, 133-151 (2000).

6. F. Ramus, G. Szenkovits, Q. J. Exp. Psychol. 61, 129-141 (2008).

7. F. Ramus, M. Ahissar, Cogn. Neuropsychol. 29, 104-122 (2012).

8. K. A. Norman, S. M. Polyn, G. J. Detre, J. V. Haxby, Trends Cogn. Sci. 10, 424-430 (2006).

9. E. Formisano, F. De Martino, M. Bonte, R. Goebel, Science 322, 970-973 (2008).

10. J. Obleser, A. M. Leaver, ]. Vanmeter, J. P. Rauschecker, Front. Psychol. 1, 232 (2010).

11. M. Vandermosten et al., Proc. Natl. Acad. Sci. U.S.A. 107, 10389-10394 (2010).

12. M. Vandermosten et al., Brain 135, 935-948 (2012).

13. B. De Smedt, B. Boets, Neuropsychologia 48 , 3973-3981 (2010).

14. H. P. Op de Beeck, K. Torfs, J. Wagemans, J. Neurosci. 28, 10111-10123 (2008).

15. R. J. Zatorre, ]. T. Gandour, Philos. Trans. R. Soc. London Ser. B 363, 1087-1104 (2008).

16. N. Kriegeskorte, R. Goebel, P. Bandettini, Proc. Natl. Acad. Sci. U.S.A. 103, 3863-3868 (2006).

17. J. F. Jehee, D. K. Brady, F. Tong, J. Neurosci. 31, 8210-8219 (2011).

18. J. Hornickel, N. Kraus, J. Neurosci. 33, 3500-3504 (2013).
19. S. Bookheimer, Annu. Rev. Neurosci. 25, 151-188 (2002).

20. S. K. Scott, I. S. Johnsrude, Trends Neurosci. 26, 100-107 (2003).

21. C. R. Gillebert, D. Mantini, Neuroscientist 19, 509-522 (2013).

22. D. A. Fair et al., Neuroimage 35, 396-405 (2007).

23. ]. M. Tyszka, D. P. Kennedy, R. Adolphs, L. K. Paul, J. Neurosci. 31, 15154-15162 (2011)

24. M. Vandermosten, B. Boets, J. Wouters, P. Ghesquière, Neurosci. Biobehav. Rev. 36, 1532-1552 (2012).

25. S. van der Mark et al., Neuroimage 54, 2426-2436 (2011).

Acknowledgments: Supported by KU Leuven (OT/07/034, IDO/10/003), Research Foundation Flanders (G0331.08, postdoctoral fellowship of B.B. and M.V.), European Research Council (grant ERC-2011-Stg-284101), Federal Research Action (IUAP/PAI P7/11), Queen Fabiola Foundation, Wellcome Trust [Sir Henry Wellcome fellowship of C.R.G. (098771/Z/12/Z), Sir Henry Dale fellowship of D.M. (101253/Z/13/Z), and grant WT090961MA of S.K.S.], and the Royal Society (D.M.: 101253/Z/13/Z). Matlab scripts are available in the supplementary materials. We thank R. Peeters and H. Poelmans for assistance. B.B., H.P.O.d.B., M.V., S.K.S., S.S., J.W., and P.G. designed the study; B.B. and M.V. collected the data; B.B. analyzed the data and wrote the manuscript; H.P.O.d.B. and J.B. contributed to MVPA analyses; M.V. and S.S. contributed to DTI analyses; C.R.G. and D.M. contributed to functional connectivity analyses; and all authors discussed the results and commented on the manuscript.

\section{Supplementary Materials}

www.sciencemag.org/content/342/6163/1251/suppl/DC1 Materials and Methods

Supplementary Text

Figs. S1 to S5

Tables $\mathrm{S} 1$ to $\mathrm{S} 4$

References (26-30)

Matlab Scripts

6 August 2013; accepted 21 October 2013 10.1126/science. 1244333

\section{MicroRNA-128 Governs Neuronal Excitability and Motor Behavior in Mice}

\begin{abstract}
Chan Lek Tan, ${ }^{{ }^{*}}$ ]oshua L. Plotkin, ${ }^{2}$ Morten T. Venø, ${ }^{3}$ Melanie von Schimmelmann, ${ }^{1,4}$ Philip Feinberg, ${ }^{1,4}$ Silas Mann, ${ }^{1,4}$ Annie Handler, ${ }^{1}$ ]ørgen Kjems, ${ }^{3}$ D. James Surmeier, ${ }^{2}$ Dónal $0^{\prime}$ Carroll, ${ }^{5,6}$ Paul Greengard, ${ }^{1}$ Anne Schaefer ${ }^{1,4} \dagger$
\end{abstract}

The control of motor behavior in animals and humans requires constant adaptation of neuronal networks to signals of various types and strengths. We found that microRNA-128 (miR-128), which is expressed in adult neurons, regulates motor behavior by modulating neuronal signaling networks and excitability. miR-128 governs motor activity by suppressing the expression of various ion channels and signaling components of the extracellular signal-regulated kinase ERK2 network that regulate neuronal excitability. In mice, a reduction of miR-128 expression in postnatal neurons causes increased motor activity and fatal epilepsy. Overexpression of miR-128 attenuates neuronal responsiveness, suppresses motor activity, and alleviates motor abnormalities associated with Parkinson's-like disease and seizures in mice. These data suggest a therapeutic potential for miR-128 in the treatment of epilepsy and movement disorders.

$\mathrm{M}$ icroRNA-128 (miR-128) is one of the most abundant and highest enriched miRNAs in the adult mouse and human brain (fig. S1A) $(1,2)$. The expression of
miR-128 in the mouse brain increases gradually during postnatal development and peaks in adulthood (fig. S1B) $(3,4)$. miR-128's expression in diverse brain regions (fig. S1C) suggests an im- portant role for this miRNA in processes that are common to many neuronal cell types.

The indication of a potent regulatory role for miR-128 in brain function came from our observation of early-onset fatal epilepsy in mice deficient in miR-128 (Fig. 1A). miR-128 is encoded by two separate genes, $m i R-128-1$ and $m i R-128-2$, on mouse chromosomes 1 and 9 (fig. S2, A and B) or human chromosomes 2 and 3, respectively. In mice, germline miR-128-2 deficiency results in an $80 \%$ reduction of miR- 128 expression in the forebrain, whereas ablation of the miR-128-1 gene eliminates only $20 \%$ of miR-128 (fig. S2, A and

${ }^{1}$ Laboratory of Molecular and Cellular Neuroscience, The Rockefeller University, New York, NY 10065, USA. ²Department of Physiology, Feinberg School of Medicine, Northwestern University, Chicago, IL 60611, USA. ${ }^{3}$ Interdisciplinary Nanoscience Center (iNANO) and Department of Molecular Biology and Genetics, Faculty of Science, Aarhus University, DK-8000 Aarhus, Denmark. ${ }^{4}$ Fishberg Department of Neuroscience, Department of Psychiatry, The Friedman Brain Institute, Icahn School of Medicine at Mount Sinai, New York, NY 10029, USA. ${ }^{5}$ European Molecular Biology Laboratory, Mouse Biology Unit, Via Ramarini 32, Monterotondo Scalo 00015, Italy. 'Laboratory of Immune Cell Epigenetics and Signaling, The Rockefeller University, New York, NY 10065, USA. *Present address: Department of Physiology, University of California, San Francisco, CA 94143, USA.

†Corresponding author. E-mail: anne.schaefer@mssm.edu 